\title{
Hepatitis C in Liver Allograft Recipients: Utility of One-Year Post-Transplantation Biopsy as an Indicator of Antiviral Therapy
}

\author{
Shahid Habib ${ }^{\mathrm{a}}$, Shahid Malik ${ }^{\mathrm{b}}$, Bo Fu ${ }^{\mathrm{c}}$, Joyce Chang ${ }^{\mathrm{d}}$, Michael Nalesnik ${ }^{\mathrm{e}}$, \\ Abhinav Humarf, Obaid S. Shaikh ${ }^{\mathrm{b}, \mathrm{g}}$
}

\begin{abstract}
Background: All-oral interferon-free regimens for hepatitis $\mathrm{C}$ viral (HCV) infection are highly efficacious; however, high cost is a barrier to applicability. Liver allograft recipients are particularly likely to benefit from therapy as HCV often leads to graft dysfunction and loss. In this study, we aimed to establish the utility of allograft biopsy at 1 year post-transplant as an indicator of treatment.
\end{abstract}

Methods and Results: Among 252 liver recipients enrolled, 136 (54\%) developed severe disease (fibrosing cholestatic hepatitis ( $\mathrm{FCH})$ or fibrosis stage $\geq 2$ at 1 year post-transplant). Multivariable analysis revealed younger recipient age and female gender, older donor age and $\mathrm{T}$ cell depletive therapy to be independent predictors of severe disease. Recipients with severe disease had higher rate of further graft loss compared to those with mild disease. Patients with mild disease and sustained virologic response (SVR) had the best survival rate, whereas those with severe disease and viremia had the worst survival (96\% versus $63 \%$ at 5 years).

Conclusion: In conclusion, allograft biopsy at 1 year helps identify recipients at high risk of further graft dysfunction and loss. In view of high cost of therapy, treatment should be preferably directed to highrisk patients including those with $\mathrm{FCH}$ or fibrosis stage $\geq 2$ by 1 year post-transplant.

Manuscript accepted for publication December 15, 2015

${ }^{a}$ Division of Gastroenterology \& Hepatology, Liver Research Institute, University of Arizona, Tucson, AZ, USA

${ }^{b}$ Division of Gastroenterology, Hepatology \& Nutrition, University of Pittsburgh School of Medicine, Pittsburgh, PA, USA

'Department of Biostatistics, Graduate School of Public Health, University of Pittsburgh, Pittsburgh, PA, USA

${ }^{d}$ Division of General Internal Medicine, University of Pittsburgh School of Medicine, Pittsburgh, PA, USA

'Division of Transplantation Pathology, University of Pittsburgh School of Medicine, Pittsburgh, PA, USA

fDivision of Transplantation Surgery, University of Pittsburgh School of Medicine, Pittsburgh, PA, USA

${ }^{\text {g} C o r r e s p o n d i n g ~ A u t h o r: ~ O b a i d ~ S . ~ S h a i k h, ~ U n i v e r s i t y ~ o f ~ P i t t s b u r g h ~ M e d i c a l ~}$ Center, Kaufmann Building, Suite 916, 3471 Fifth Avenue, Pittsburgh, PA 15213, USA.Email: obaid@pitt.edu

doi: http://dx.doi.org/10.14740/gr694w
Keywords: Antiviral agent; Hepatitis C; Immunosuppression; Liver fibrosis; Transplantation

\section{Introduction}

Among adult patients who undergo liver transplantation in the United States, $23 \%$ have hepatitis C virus (HCV) infection (Organ Procurement and Transplantation Network; http://optn. transplant.hrsa.gov; accessed July 2014). Hepatitis C is notable as a cause of morbidity in liver transplant recipients that results from universal recurrence of infection and an accelerated disease course. Thus, HCV-positive recipients tend to have inferior graft and patient survival compared to $\mathrm{HCV}$-negative recipients [1]. The severity of post-transplant hepatitis $\mathrm{C}$ has a wide spectrum that ranges from minimal inflammatory activity without fibrosis to progressive graft fibrosis and graft failure to rapid and early graft failure from fibrosing cholestatic hepatitis (FCH) [2]. Certain features have been identified to be associated with inferior outcomes. Among them donor age, pretransplant viral load, acute rejection episodes and use of bolus corticosteroids and $\mathrm{T}$ cell depletive therapy have been found to be particularly influential $[3,4]$.

Highly effective interferon-free regimens, incorporating NS5A, NS5B and protease inhibitors with or without ribavirin, are now the standard of care for HCV genotypes 1 and 4. For genotypes 2 and 3, sofosbuvir, an NS5B inhibitor, with ribavirin is recommended. The safety and efficacy of interferon-free regimens in transplant recipients remain under investigation [5]. Current regimens have little interaction with calcineurin inhibitors, which was prominent with first-generation protease inhibitors [6]. However, as ribavirin is likely needed in this difficult to treat, immunosuppressed population, anemia will remain a consideration during therapy [7]. The use of all-oral regimens has been hampered by prohibitive cost particularly in low income countries $[8,9]$. Transplant recipients with HCV form a special population where viral eradication is highly desirable in view of accelerated disease course and risk of graft failure. Considering limited resources in many parts of the world, it will be prudent to prioritize therapy to recipients with severe disease and/or to those likely to develop severe disease. Patients with mild disease could be followed closely and treated when more affordable regimens become available or 


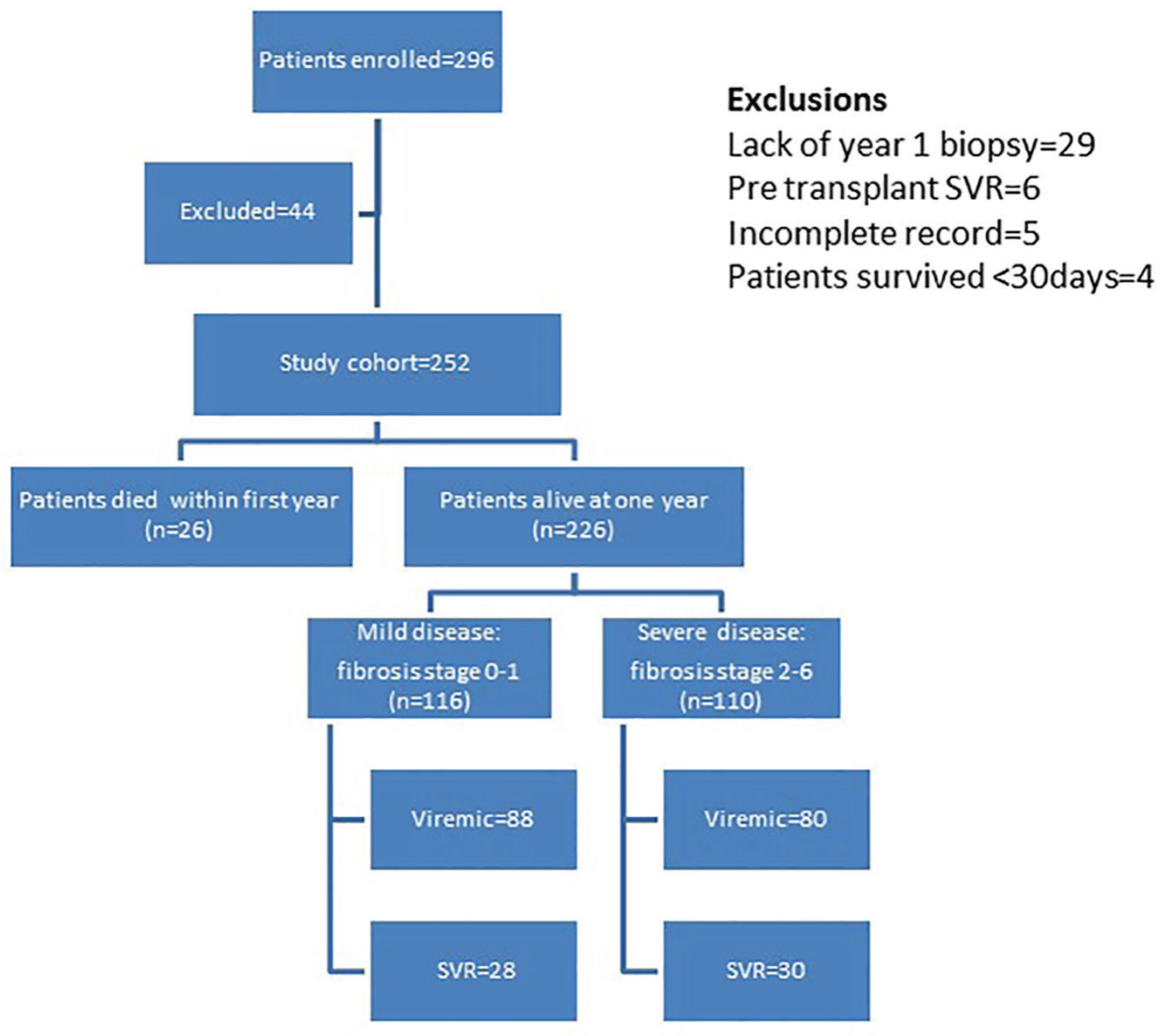

Figure 1. Distribution of patients in study cohort.

if their disease progresses. One approach is to base treatment decisions on protocol allograft biopsies as liver pathologies at 4 months and at 1 year of transplantation have been shown to differentiate subsequent slow or rapid progression of $\mathrm{HCV}$ disease $[10,11]$.

The aim of our study was to establish the utility of allograft biopsy at 1 year post-transplant as a determinant of further progression of disease. We hypothesized that the development of FCH or fibrosis stage $\geq 2$ at 1 year post-transplant was associated with rapid progression of disease leading to early graft failure. Such patients would therefore benefit from effective antiviral treatment causing viral clearance. Our secondary goals were to determine factors that influenced disease progression and to determine the impact of sustained virologic response (SVR) to antiviral therapy on long-term allograft and patient survival.

\section{Patients and Methods}

\section{Patient enrollment}

We enrolled adult, HCV-positive liver transplant recipients in a prospective follow-up cohort study. The study required subjects to be anti-HCV positive, have detectable HCV RNA and hepatitis B surface antigen (HBsAg) and antibody to hu- man immunodeficiency virus (HIV) negative. Recipients who died within 1 month of transplantation were excluded from the current study. Once a patient was identified to be a suitable subject, a research coordinator introduced the study to the patient and provided details of study participation. Subjects were recruited in the clinic that provided longitudinal care to liver transplant patients. Follow-up visits were scheduled at $3-6$ monthly intervals or more frequently if indicated. All participants signed an Institutional Review Board (IRB) approved consent form.

\section{Patient evaluation}

At the initial evaluation, a complete history and examination were obtained. In addition, laboratory, radiologic and pathologic features were noted, and Child-Pugh and model for endstage liver disease (MELD) scores were calculated. Donor and operative variables studied were age, gender, race, live or deceased, mismatch for gender, race and blood group, cold and warm ischemia time, operative technique and transfusion requirements. Immunosuppressive regimen, post-transplant course and complications were also recorded. Protocol allograft biopsies were obtained at 1 year following transplantation and every 3 years, subsequently. Additional biopsies were obtained as clinically indicated. All biopsies were read by the study pathologist. Hepatic inflammatory activity and fibrosis 
Table 1. Demographics and Baseline Clinical Features

\begin{tabular}{|c|c|c|c|}
\hline & Mild disease $(n=116)$ & Severe disease $(n=136)$ & Pvalue \\
\hline Age (years) & $52 \pm 7$ & $51 \pm 6$ & 0.06 \\
\hline Gender (male) & $98(84 \%)$ & $96(71 \%)$ & 0.009 \\
\hline Caucasian & $107(92 \%)$ & $124(91 \%)$ & 0.76 \\
\hline Child-Pugh status & & & 0.14 \\
\hline Class A & $12(10 \%)$ & $6(4 \%)$ & \\
\hline Class B & $69(59 \%)$ & $80(59 \%)$ & \\
\hline Class C & $35(30 \%)$ & $50(37 \%)$ & \\
\hline Child-Pugh score & $9 \pm 1.8$ & $9 \pm 1.6$ & 0.12 \\
\hline MELD score & $16 \pm 7$ & $16 \pm 8$ & 0.87 \\
\hline Blood group & & & 0.62 \\
\hline A & $40(34 \%)$ & $56(41 \%)$ & \\
\hline B & $11(9 \%)$ & $14(10 \%)$ & \\
\hline $\mathrm{AB}$ & $9(8 \%)$ & $7(5 \%)$ & \\
\hline $\mathrm{O}$ & $56(48 \%)$ & $59(43 \%)$ & \\
\hline \multicolumn{4}{|l|}{ Co-morbidities } \\
\hline Diabetes mellitus & $35(30 \%)$ & $37(27 \%)$ & 0.60 \\
\hline Hypertension & $43(37 \%)$ & $43(32 \%)$ & 0.36 \\
\hline BMI $\left(\mathrm{kg} / \mathrm{m}^{2}\right)$ & $29 \pm 5$ & $28 \pm 5$ & 0.12 \\
\hline Obesity (BMI $\geq 30)$ & $44(38 \%)$ & $43(32 \%)$ & 0.29 \\
\hline Renal Failure & $16(14 \%)$ & $22(16 \%)$ & 0.60 \\
\hline Hepatocellular carcinoma & $32(28 \%)$ & $37(27 \%)$ & 0.95 \\
\hline
\end{tabular}

All values are shown as mean \pm SD or proportion as appropriate. Comparison by two-tailed $t$-test or Chi-square test as appropriate. n: number. SD: standard deviation.

were graded and staged according to the Ishak's histology activity index [12], and graft rejection was graded according to the Banff schema [10]. If a graft was lost within 4 weeks of transplantation, we included the second graft instead. Ten milliliter of whole blood was obtained at each visit and separated serum was stored at $-80^{\circ} \mathrm{C}$ for later studies.

\section{Anti-HCV therapy}

We initiated antiviral therapy if a patient developed FCH or if fibrosis stage was $\geq 2$ at 1 year post-transplant. Treatment was otherwise deferred until criteria were met on subsequent evaluations. The decision to treat patients with stage $\geq 2$ fibrosis was based on our understanding that such patients had high likelihood of progression to graft failure and death [11]. Conversely, recipients with lesser degree of fibrosis had low risk of disease progression and graft failure. In low-risk group, treatment was offered to those who requested treatment, regardless of histologic severity. Patients noted to have negative HCV RNA 6 months after discontinuation of treatment were considered to have SVR. Patients who received antiviral treatment and either did not respond or relapsed after discontinuation of therapy were considered viremic. The latter group also included those who did not receive antiviral therapy.

\section{Study endpoints}

The primary endpoint was cumulative graft and patient survival among patients alive at 1 year post-transplant. Recipients categorized to have mild (stage 0 - 1 fibrosis) versus severe (stage 2 - 6 fibrosis or FCH) disease were compared, and factors predictive of graft loss were determined. In addition, we determined factors that predicted development of severe disease by 1 year post-transplant.

\section{Statistical analysis}

We evaluated recipient, donor and operative variables at the time of transplantation and compared patients categorized to have mild versus severe disease at 1 year post-transplant. Continuous variables were assessed by the Student's $t$-test and categorical variables by Chi-square test with Yates continuity correction if applicable. Logistic regression analysis was used to determine factors predictive of disease severity as described. 
Table 2. Baseline Laboratory Features

\begin{tabular}{|c|c|c|c|}
\hline & Mild disease $(n=116)$ & Severe disease $(n=136)$ & P value \\
\hline Hemoglobin (g/dL) & $12.0 \pm 2.1$ & $11.7 \pm 2.1$ & 0.20 \\
\hline $\mathrm{WBC}\left(\times 10^{3} / \mathrm{L}\right)$ & $5.0 \pm 2.7$ & $5.9 \pm 4.0$ & 0.04 \\
\hline Platelets $\left(\times 10^{3} / \mathrm{L}\right)$ & $67 \pm 40$ & $74 \pm 50$ & 0.25 \\
\hline Bilirubin (mg/dL) & $4.1 \pm 6.2$ & $4.6 \pm 7.4$ & 0.55 \\
\hline $\operatorname{ALT}(\mathrm{IU} / \mathrm{L})$ & $131 \pm 411$ & $152 \pm 420$ & 0.69 \\
\hline AST (IU/L) & $334 \pm 1926$ & $255 \pm 914$ & 0.67 \\
\hline $\operatorname{ALP}(\mathrm{IU} / \mathrm{L})$ & $166 \pm 106$ & $164 \pm 88$ & 0.87 \\
\hline$\gamma \mathrm{GTP}(\mathrm{IU} / \mathrm{L})$ & $97 \pm 116$ & $95 \pm 97$ & 0.89 \\
\hline Albumin $(\mathrm{g} / \mathrm{dL})$ & $2.9 \pm 0.5$ & $2.8 \pm 0.6$ & 0.29 \\
\hline INR & $1.4 \pm 0.4$ & $1.4 \pm 0.5$ & 0.95 \\
\hline Creatinine (mg/dL) & $1.3 \pm 1.3$ & $1.4 \pm 1.4$ & 0.77 \\
\hline $\operatorname{HCV} \operatorname{RNA}\left(\times 10^{6} \mathrm{IU} / \mathrm{mL}\right)^{*}$ & $1.02 \pm 3.4$ & $2.0 \pm 6.4$ & 0.16 \\
\hline HCV genotype $1 * *$ & $89(81 \%)$ & $111(87 \%)$ & 0.22 \\
\hline Anti-CMV, IgG (IU/L) & $105 \pm 122$ & $102 \pm 126$ & 0.86 \\
\hline Anti-CMV, IgG-positive*** & $81(70 \%)$ & $81(60 \%)$ & 0.09 \\
\hline Anti-EBV, IgG (IU/L) & $251 \pm 222$ & $242 \pm 196$ & 0.75 \\
\hline Anti-EBV, IgG-positive**** & $79(71 \%)$ & $101(77 \%)$ & 0.24 \\
\hline
\end{tabular}

All values are shown as mean \pm SD or proportion as appropriate. Comparison by two-tailed $t$-test or Chi-square test as appropriate. ${ }^{*} n=209 .{ }^{* *} n=238$; data unavailable in 14 patients (mild disease: 5 , severe disease: 9 ). ${ }^{* * *}$ Cytomegalovirus antibody (positive: > 4 IU/L). ${ }^{* \star * *}$ Epstein-Barr viral capsid antigen antibody (positive: > 20 IU/L); $n=243$. n: number. SD: standard deviation; IU: international units.

Table 3. Donor Features

\begin{tabular}{|c|c|c|c|}
\hline & Mild disease $(n=116)$ & Severe disease $(n=136)$ & P value \\
\hline Age (years) & $41 \pm 17$ & $45 \pm 16$ & 0.026 \\
\hline Males & $68(59 \%)$ & $78(57 \%)$ & 0.84 \\
\hline Caucasian & $96(83 \%)$ & $120(88 \%)$ & 0.21 \\
\hline Living donor & $9(8 \%)$ & $14(10 \%)$ & 0.49 \\
\hline Anti-HCV positive* & $13(11 \%)$ & $22(17 \%)$ & 0.25 \\
\hline Anti-HBc positive** & $13(12 \%)$ & $14(11 \%)$ & 0.86 \\
\hline Anti-CMV IgG positive*** & $70(63 \%)$ & $82(61 \%)$ & 0.76 \\
\hline Donor biopsy & $61(53 \%)$ & $82(60 \%)$ & 0.22 \\
\hline Blood group & & & 0.83 \\
\hline A & $39(34 \%)$ & $52(38 \%)$ & \\
\hline B & $13(11 \%)$ & $13(10 \%)$ & \\
\hline $\mathrm{AB}$ & $7(6 \%)$ & $6(4 \%)$ & \\
\hline $\mathrm{O}$ & $57(49 \%)$ & $65(48 \%)$ & \\
\hline \multicolumn{4}{|l|}{ Donor recipient mismatch } \\
\hline Gender & $48(41 \%)$ & $58(43 \%)$ & 0.84 \\
\hline Racial & $24(21 \%)$ & $26(19 \%)$ & 0.75 \\
\hline ABO blood group & $5(4 \%)$ & $7(5 \%)$ & 0.76 \\
\hline
\end{tabular}

All values are shown as mean \pm SD or proportion as appropriate. Comparison by two-tailed $t$-test or Chi-square test as appropriate. ${ }^{*} n=247$. ${ }^{* *} n=243$. ${ }^{* * *} n=245$. $n$ : number. SD: standard deviation; anti-HCV: antibody to hepatitis C virus; anti-HBc: antibody to hepatitis B core antigen; CMV: cytomegalovirus. 
Table 4. Operative Features

\begin{tabular}{llll}
\hline & Mild disease $(\mathbf{n}=\mathbf{1 1 6})$ & Severe disease $(\mathbf{n}=\mathbf{1 3 6})$ & P value \\
\hline Cold ischemia time $(\mathrm{h})$ & $9.8 \pm 3.4$ & $9.9 \pm 3.9$ & 0.82 \\
Warm ischemia time (min) & $34 \pm 11$ & $33 \pm 10$ & 0.24 \\
Red cell transfusion (unit)* & $7.5 \pm 6.7$ & $8.5 \pm 7.5$ & 0.28 \\
Platelets (unit) & $6.2 \pm 8.1$ & $7.7 \pm 9.4$ & 0.20 \\
Fresh frozen plasma (unit)* & $6.4 \pm 6.6$ & $7.6 \pm 7.3$ & 0.20 \\
Piggyback graft placement & $98(84 \%)$ & $117(86 \%)$ & 0.73 \\
Duct-duct anastomosis & $110(95 \%)$ & $129(95 \%)$ & 1.0 \\
T-tube placement & $77(66 \%)$ & $96(71 \%)$ & 0.47 \\
Veno-venous bypass & $81(70 \%)$ & $94(69 \%)$ & 0.90 \\
$\begin{array}{l}\text { Length of stay post- } \\
\text { transplant (days) }\end{array}$ & $19 \pm 19$ & $21 \pm 23$ & 0.64 \\
\hline
\end{tabular}

All values are shown as mean \pm SD or proportion as appropriate. Comparison by two-tailed $t$-test or Chi-square test as appropriate. ${ }^{*} n=246 . n$ : number. SD: standard deviation.

Cumulative survival was determined by Kaplan-Meier analysis and the two severity groups were compared by log-rank test. Factors predictive of long-term survival were determined by Cox proportional hazards modeling. All analyses were performed using $\mathrm{SAS}^{\mathrm{TM}} 9.2$ and $\mathrm{R}^{\mathrm{TM}} 2.12 .1$.

\section{Results}

\section{Study cohort}

Figure 1 outlines the distribution of the study cohort. Two hun- dred ninety-six liver transplant recipients were enrolled over a period of 8 years (2002 - 2009). Forty-four recipients were excluded from this study for the following reasons: 29 did not have 1 year allograft biopsy, six were HCV RNA negative pre- and post-transplant, five had incomplete information and four survived $<30$ days post-transplant; thus, 252 recipients were studied. Based on 1 year post-transplant histologic evaluation and outcome, 116 recipients were categorized to have mild disease (fibrosis stage 0 - 1) and 136 were categorized to have severe disease ( 110 with fibrosis stage 2 - 6, 26 with posttransplant survival of $<1$ year). Patients were followed for a mean follow-up of 5.6 years $(\mathrm{SD} \pm 2.8)$.

The mean age of the cohort was 51 years and $77 \%$ were

Table 5. Immunosuppression and Antiviral Therapy

\begin{tabular}{llll}
\hline & Mild disease $(\mathbf{n}=\mathbf{1 1 6})$ & Severe disease $(\mathbf{n = 1 3 6})$ & P value \\
\hline Corticosteroids & $107(92 \%)$ & $121(89 \%)$ & 0.38 \\
Anti-T cell antibody* & $10(9 \%)$ & $27(20 \%)$ & 0.012 \\
Mycophenolate mofetil & $29(25 \%)$ & $33(24 \%)$ & 0.89 \\
Sirolimus & $1(1 \%)$ & $4(3 \%)$ & 0.24 \\
Acute rejection & $33(28 \%)$ & $60(44 \%)$ & 0.01 \\
Acute rejection episodes & $0.4 \pm 0.7$ & $0.6 \pm 0.8$ & 0.034 \\
ACR: steroid boluses & $29(25 \%)$ & $50(37 \%)$ & 0.045 \\
Chronic rejection & $4(3 \%)$ & $9(7 \%)$ & 0.26 \\
Cumulative steroid dose $(\mathrm{g}) * *$ & $154 \pm 780$ & $214 \pm 781$ & 0.54 \\
Cumulative tacrolimus & $467 \pm 577$ & $686 \pm 996$ & 0.038 \\
dose (mg/year) & & $99(73 \%)$ & 0.41 \\
Interferon therapy*** & $79(68 \%)$ & $31(23 \%)$ & 0.8 \\
\hline Interferon responders & $28(24 \%)$ & & \\
\hline
\end{tabular}

All values are shown as mean \pm SD or proportion as appropriate. Comparison by two-tailed $t$-test or Chi-square test as appropriate. $\mathrm{n}$ : number. SD: standard deviation. ${ }^{*}$ Perioperative with either antithymocyte globulin or alemtuzumab. ${ }^{* *}$ During the first post-operative year and hydrocortisone equivalent. ${ }^{* * *}$ Post-transplant treatment with pegylated interferon- $\alpha 2 b$ or pegylated interferon- $\alpha 2 a$ with ribavirin. 
Table 6. Predictors of Disease Severity at 1 Year Post-Transplant

\begin{tabular}{lllll}
\hline Variables included & $\mathbf{B} \pm \mathbf{S E}$ & Odds ratio & $\mathbf{9 5 \%}$ CI & P value \\
\hline Recipient age & $-0.055 \pm 0.02$ & 0.95 & $0.91-0.98$ & 0.007 \\
Female gender & $0.96 \pm 0.34$ & 2.6 & $1.33-5.07$ & 0.005 \\
Donor age & $0.02 \pm 0.01$ & 1.02 & $1.0-1.04$ & 0.021 \\
Anti-T cell antibody & $0.85 \pm 0.41$ & 0.43 & $0.19-0.95$ & 0.037 \\
Constant & $2.7 \pm 1.12$ & 14.88 & & 0.016 \\
\hline
\end{tabular}

Logistic regression analysis. $R^{2}=0.07$ (Hosmer \& Lemeshow), 0.09 (Cox \& Snell), 0.12 (Nagelkerke). Model $X^{2}=24.1, P<0.001$. B: coefficient of regression. SE: standard error; $\mathrm{Cl}$ : confidence interval.

men. More than $90 \%$ were Caucasian, they largely had Child's $\mathrm{B}$ or $\mathrm{C}$ disease and their mean MELD score at transplantation was 16 . Almost one-third had co-morbidities including obesity (34\%), hypertension (34\%), diabetes mellitus (29\%) and renal failure $(15 \%)$. Known or incidental hepatocellular carcinoma was noted in $27 \%$ of the recipients. Among patients with available HCV RNA level, 32\% had viral load of $>850,000$ international units per $\mathrm{mL}(\mathrm{IU} / \mathrm{mL})$ and $84 \%$ had $\mathrm{HCV}$ genotype 1. Majority were seropositive for cytomegalovirus (CMV) and Epstein-Barr virus. Fourteen percent of donors were anti$\mathrm{HCV}$ positive, $11 \%$ were antibody to hepatitis B core antigen (anti-HBc) positive and 62\% were seropositive for CMV. Tacrolimus was the primary immunosuppressant and in addition, one-quarter received mycophenolate. Most patients were given corticosteroids, typically intravenous methylprednisone followed by oral prednisone that was generally weaned off within 6 months. About 15\% received lymphocyte depletive therapy with either anti-thymocyte globulin or alemtuzumab, during the peri-operative and/or immediate post-operative phase.

\section{Factors related to severity of disease during first year of transplantation}

\section{Univariable analysis}

One hundred thirty-six patients (54\%) developed severe disease within first year of transplantation (Tables 1-5). Factors significantly associated with severe disease included female gender, high white cell count and older donor age; however, no particular age threshold was noted. We examined several operative features including cold and warm ischemia time and the use of blood products (red blood cells, platelets and fresh frozen plasma). In addition, we looked at operative techniques, choledocho-choledocho anastomosis versus choledocho-jejunostomy, veno-venous bypass, graft placement in a piggyback fashion with inferior venacava preservation versus standard implantation and length of hospital stay. None of those features was significantly different among the two groups. Pa-

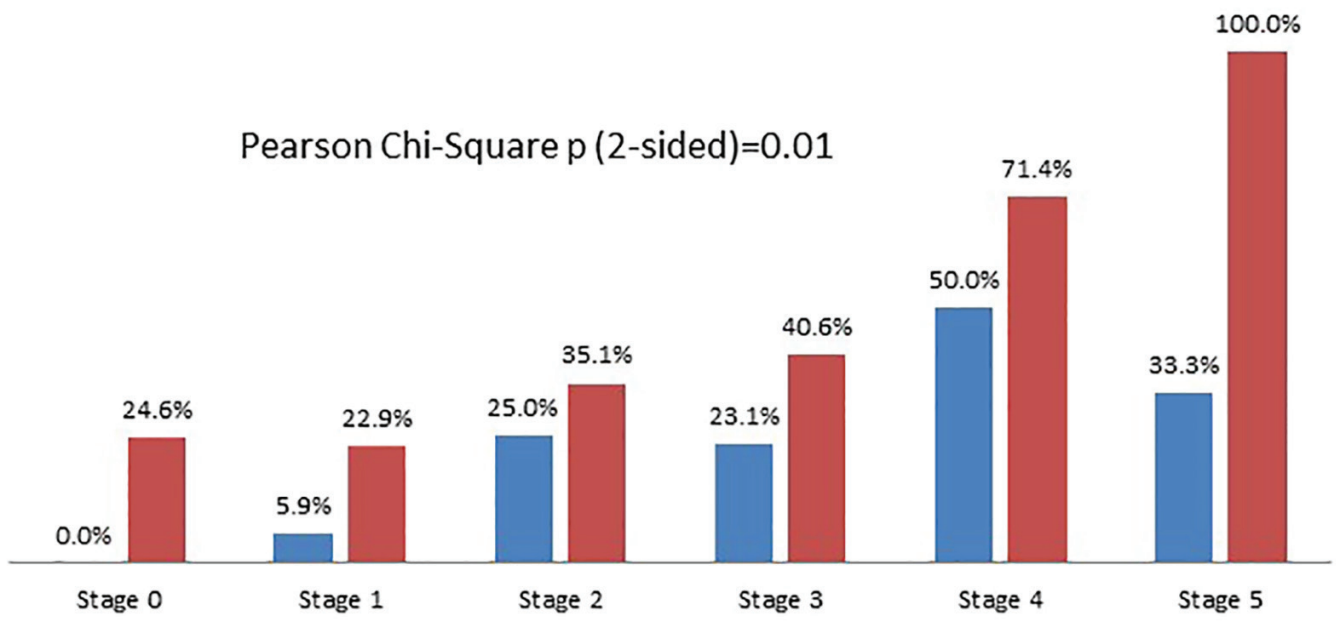

Fibrosis stage at 1 year post transplantation

Figure 2. Graft survival rate in SVR and viremic patients categorized according to fibrosis stage at 1 year post-transplant. There is a trend of worsening graft failure from stage 0 to stage 5 . 
Table 7. Prediction of Graft Failure Beyond First Post-Transplant Year

\begin{tabular}{llll}
\hline Variable & $\mathbf{B} \pm \mathbf{S E}$ & Hazard ratio & P value \\
\hline Fibrosis stage 5/6 & $1.24 \pm 0.51$ & 3.46 & 0.016 \\
Fibrosis stage 3/4 & $0.81 \pm 0.32$ & 2.25 & 0.011 \\
Fibrosis stage 2 & $0.54 \pm 0.36$ & 1.71 & 0.133 \\
Recipient age & $0.01 \pm 0.02$ & 1.01 & 0.649 \\
Female gender & $-0.13 \pm 0.32$ & 0.88 & 0.690 \\
Donor age & $0.03 \pm 0.01$ & 1.03 & 0.0007 \\
Anti-T cell antibody & $0.32 \pm 0.30$ & 1.38 & 0.285 \\
\hline
\end{tabular}

Cox proportional hazards analysis. B: coefficient of regression. SE: standard error.

tients with severe disease were more likely to have received $\mathrm{T}$ cell depletive therapy, had a higher likelihood of acute rejection episodes and of treatment with bolus corticosteroids. In addition, they received higher cumulative doses of tacrolimus per unit of time. About 5\% develop changes consistent with chronic rejection.

\section{Multivariable analysis}

We selected factors previously recognized to influence disease severity and those found significant on univariable analysis for forced entry into logistic regression (Table 6). After multiple iterations, we reached the most efficient model that included four variables. The assumptions of linearity of logit and lack of multicollinearity were tested and were noted to be fulfilled. Younger recipient age, female gender, older donor age and use of anti-T cell antibody were independently predictive of severe disease. Female gender had the highest effect size with an odds ratio of 2.6. The model was significant and explained $7-12 \%$ of variability in the likelihood of development of severe disease as indicated by the $\mathrm{R}^{2}$ values.

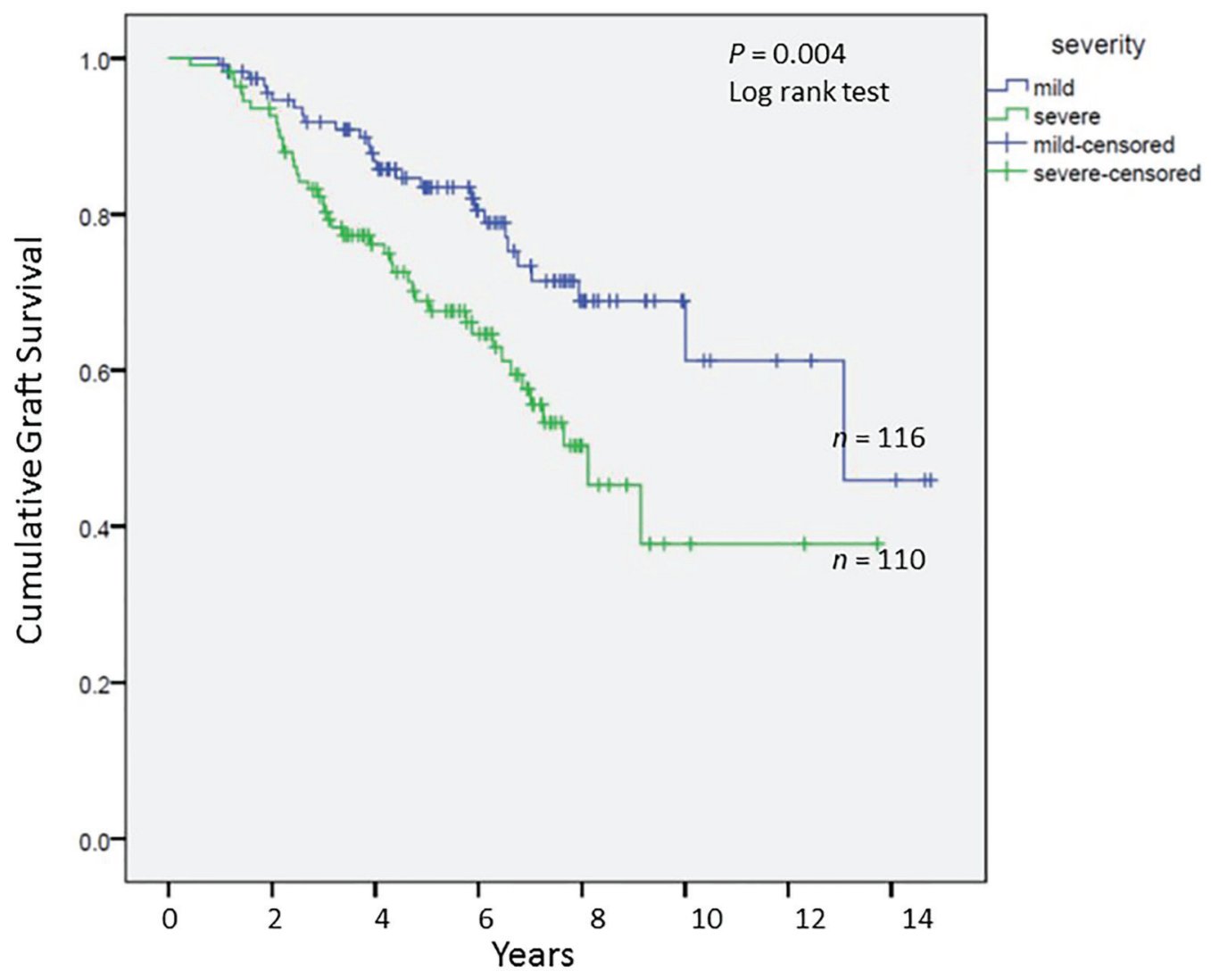

Figure 3. Cumulative graft survival among patients alive at 1 year post-transplant; Kaplan-Meier analysis, comparison by logrank test. 


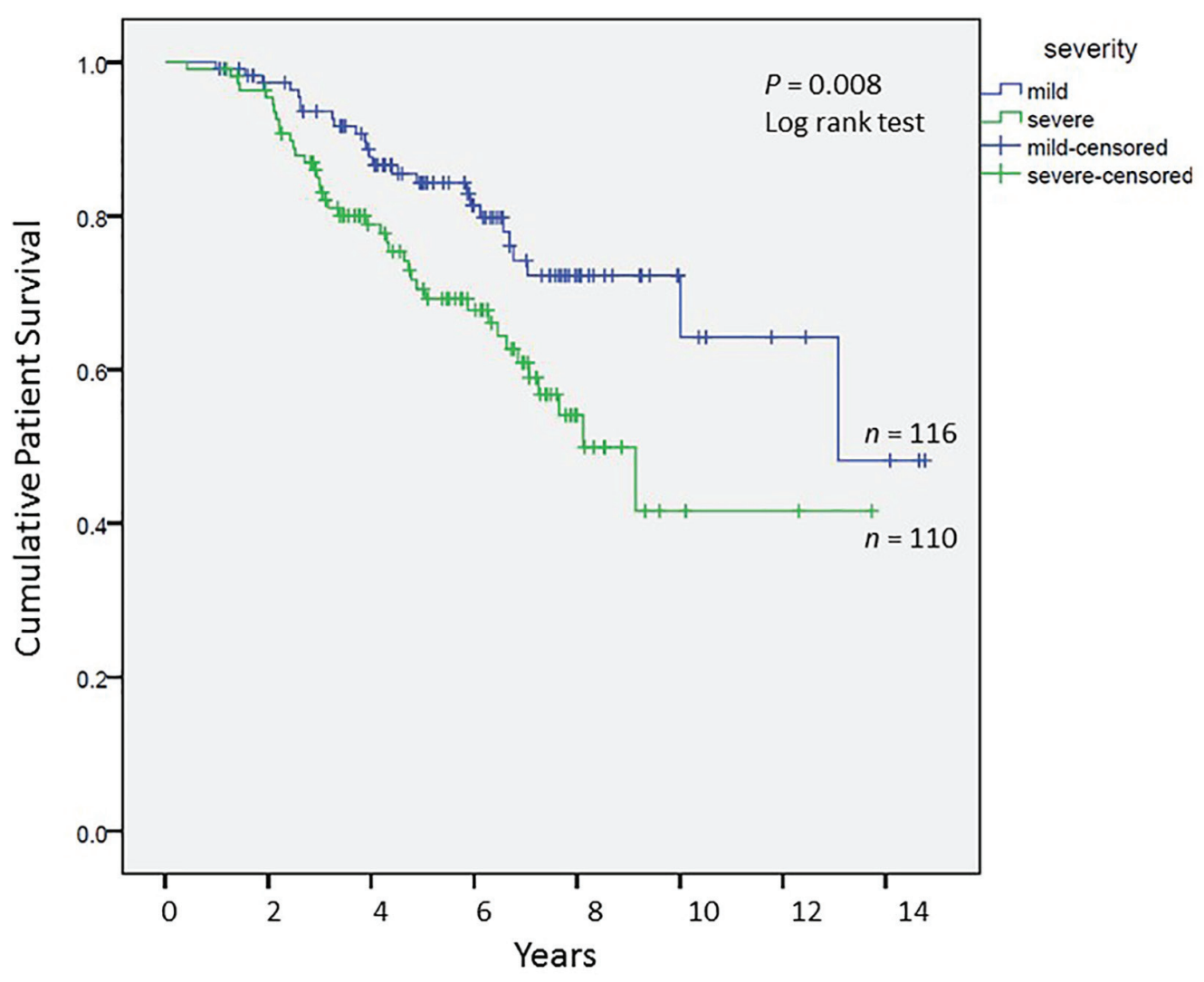

Figure 4. Cumulative patient survival among patients alive at 1 year post-transplant; Kaplan-Meier analysis, comparison by logrank test.

\section{Analysis of patients who survived 1 year post-transplan- tation}

Among 252 transplant recipients studied, $226(90 \%)$ were alive at 1 year: 116 with mild disease and 110 with severe disease. One hundred seventy-nine of the 226 (71\%) patients received pegylated interferon and ribavirin with similar proportions in the two groups. Fifty-eight of the 179 patients treated (32\%) were HCV RNA negative at 6 months following completion of treatment and thus achieved SVR with no difference between the two groups. We further categorized patients into those who had SVR versus those who remained viremic with or without therapy (Fig. 1). Figure 2 depicts distribution of allograft failure in the SVR and viremic groups according to stage of

Table 8. Allograft Failure Risk Based on Treatment Status

\begin{tabular}{lllll} 
& Pvalue & HR & \multicolumn{2}{c}{$\mathbf{9 5 . 0 \%}$ CI HR } \\
\cline { 4 - 5 } & & & Lower & Upper \\
\hline Mild disease viremic & 0.001 & & & \\
Mild disease SVR & 0.026 & 0.103 & 0.014 & 0.761 \\
Severe disease viremic & 0.007 & 2.050 & 1.218 & 3.451 \\
Severe disease SVR & 0.419 & 0.721 & 0.326 & 1.594 \\
\hline
\end{tabular}

$\mathrm{Cl}$ : confidence interval; HR: hazard ratio. allograft fibrosis at 1 year post-transplant. In both groups, there was a trend towards higher graft loss with increasing fibrosis stage at 1 year post-transplant. Nevertheless, viral eradication significantly reduced the risk of allograft failure at all fibrosis stages. In a multivariable analysis, we evaluated the effect of baseline variables and fibrosis stage at 1 year on long-term graft survival (Table 7). Beyond the first year, recipient age and gender and use of $\mathrm{T}$ cell depletive therapy had no effect on graft survival; however, donor age continued to be highly predictive. Fibrosis stage 0,1 or 2 had no influence but fibrosis stages $3 / 4$ and $5 / 6$ predicted inferior graft survival with hazard ratios of 2.25 and 3.46 , respectively.

\section{Long-term graft and patient survival}

We analyzed long-term graft and patient survival among patients alive at 1 year post-transplant, with recipients categorized to have mild versus severe disease as defined earlier (Fig. $3,4)$. Among those alive at 1 year, the mean duration of graft survival was $9.9( \pm 0.5)$ years, with 3-and 5-year survival of $92 \%$ and $84 \%$ in those with mild disease and $81 \%$ and $69 \%$ in those with severe disease $(\mathrm{P}=0.004)$. The mean duration of patient survival was $10.3( \pm 0.5)$ years, with 3 -and 5 -year survival of $94 \%$ and $84 \%$ in those with mild disease and $84 \%$ and $70 \%$ in those with severe disease $(\mathrm{P}=0.008)$. We performed 


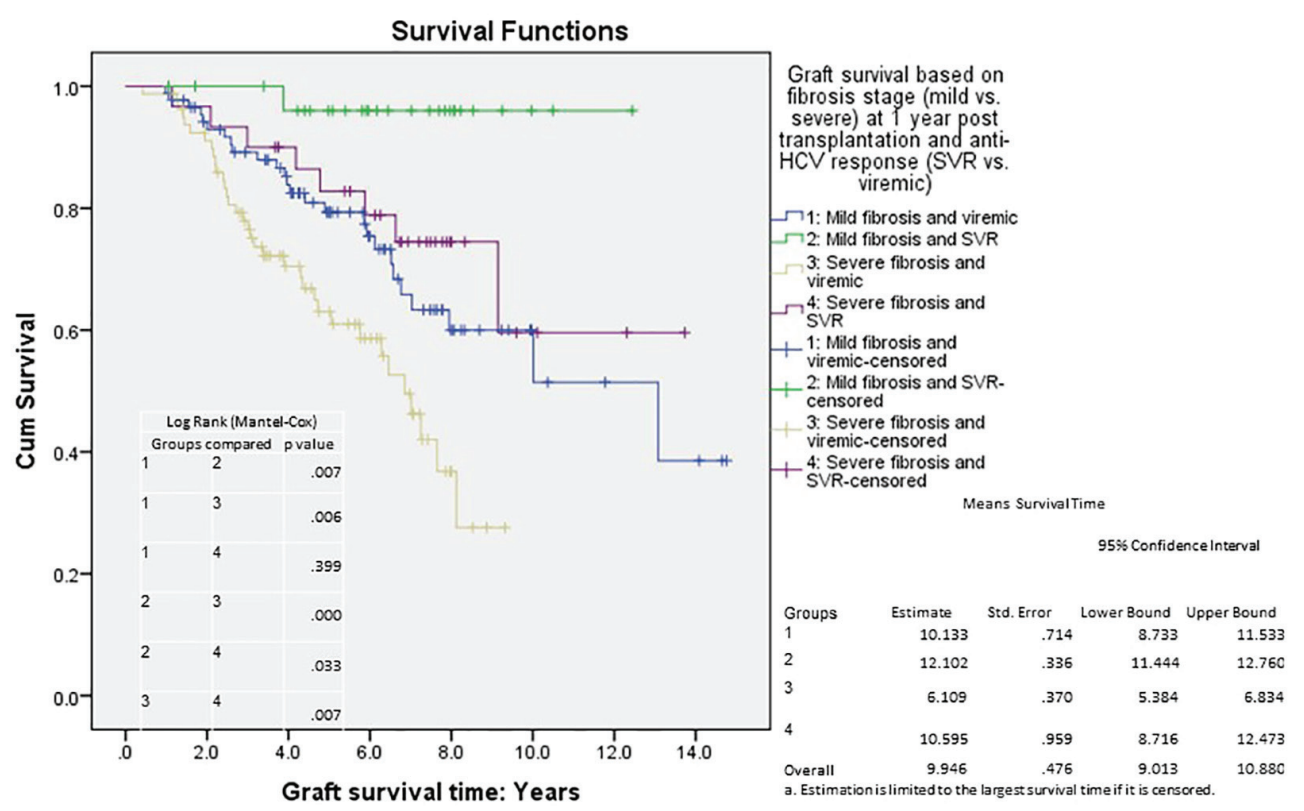

Figure 5. Cumulative graft survival according to fibrosis stage (at 1 year post-transplant) and treatment response; Kaplan-Meier analysis, comparison by log-rank test; mean survival with confidence intervals shown. Std. error: standard error.

Cox hazard model and Kaplan-Meier analysis to evaluate impact of disease severity and viral eradication on graft survival (Table 8, Fig. 5). Compared to patients with mild disease who remained viremic, those with mild disease and SVR had significantly better graft survival whereas survival among those with severe disease and SVR was no different. Patients with severe disease who remained viremic had the worst graft survival of $63 \%$ at 5 years following transplant.

\section{Discussion}

With recent advances in antiviral therapy, the outlook for liver allograft recipients with hepatitis $\mathrm{C}$ is expected to change. A significant proportion of recipients with HCV develop graft failure either due to severe cholestatic hepatitis or from rapid progression of fibrosis [13]. Viral eradication may alter disease course resulting in improved graft and patient survival. The risk of allograft cirrhosis among recipients who survive the first year post-transplant is about $25 \%$ over a period of $5-10$ years, which suggests that the disease may remain indolent despite persistent infection [11]. Protocol liver biopsies may help identify patients at high risk of disease progression and graft failure [14]. Our study assessed the utility of allograft fibrosis stage at 1 year as an indication for antiviral therapy. Results indicated that patients with mild disease (stage 0 - 1) were at low risk of graft failure whereas those with severe disease (stage 2 - 6) were at higher risk of graft loss. On analyzing the effect of individual fibrosis stages, maximal effect leading to poor outcome was associated with stages 3 - 6 (Table 7).

All-oral interferon-free regimens are now the standard of care for hepatitis C [15]. However, prohibitive cost and limited availability in many parts of the world are barriers to their use
$[8,16,17]$. Although not approved for use in transplant recipients, preliminary studies have shown high efficacy and tolerability of such regimens [5]. Transplant recipients constitute a special population that would derive high level of benefit from viral eradication; however, not all patients have severe disease. Thus, treatment may be reserved for patients at risk of rapid progression and graft failure to optimize use of limited financial resources. Conversely, recipients with mild disease could be carefully monitored without treatment until therapies become more affordable. In our current analysis, we attempted to identify recipients who would benefit from current treatment regimens versus those who could be followed without therapy.

We identified factors that predicted development of severe disease during the first post-transplant year. By univariable analyses, patients with mild versus severe disease differed in recipient gender, pre-transplant white cell count, donor age, acute rejection episodes, $\mathrm{T}$ cell depletive therapy, use of bolus corticosteroids and cumulative tarcolimus dose. By logistic regression analysis, recipient age and gender, donor age and use of anti-T cell antibody were independently predictive of severe disease. Most of those are well recognized predictive variables in HCV-positive recipients [11]. An interaction between gender and HCV status has been noted before. In an analysis of UNOS database, female recipients with HCV infection were noted to have significantly worse graft and patient survival compared to female recipients without $\mathrm{HCV}$ infection [1]. An effect of younger recipient age has not been noted before and therefore requires confirmation. One reason for this variance may be the endpoint in this part of our study that differed from other published reports: 1 year post-transplant versus cumulative long-term observation. Our study confirmed the deleterious effect of T cell depletive therapy in HCV-positive recipients possibly related to selection of resistant T-cell clones [4]. 
We also confirmed accelerated HCV disease course in older donor allografts that suggested an adverse effect of liver senescence $[18,19]$. Still, it is difficult to include donor age in the transplant decision making process in view of the continued gap between organ availability and demand [20]. A policy to decline older organs for hepatitis $\mathrm{C}$ recipients may cause inordinate delays in transplanting such patients.

In conclusion, a significant proportion of liver transplant recipients with hepatitis $\mathrm{C}$ are at risk of allograft dysfunction and failure. Such patients could be identified by allograft biopsy at 1 year post-transplant. Recipients at high risk including those with $\mathrm{FCH}$ and those with moderate allograft fibrosis at 1 year post-transplant should be promptly initiated on antiviral therapy. Conversely, treatment may be deferred among recipients with mild disease until wider availability of more affordable and cost effective agents.

\section{Acknowledgement}

We are grateful to the transplant coordinators, research coordinators and other staff of the Starzl Transplant Institute, University of Pittsburgh, for helping us complete this study. We are also grateful to Audrianna Judd for helping coordinating publication process.

\section{Funding Sources}

None.

\section{Disclosure}

This study was approved by the Institutional Review Board of the University of Pittsburgh. It was performed in accordance with the ethical standards laid down in the 1964 Declaration of Helsinki. All subjects gave informed consent prior to their inclusion in the study.

\section{Abbreviations}

Anti-HBc: hepatitis B core antigen; CMV: cytomegalovirus; FCH: fibrosing cholestatic hepatitis; HBsAg: hepatitis B surface antigen; HCV: hepatitis $\mathrm{C}$ virus; HIV: human immunodeficiency virus; MELD: model for end-stage liver disease; SVR: sustained virologic response

\section{References}

1. Forman LM, Lewis JD, Berlin JA, Feldman HI, Lucey MR. The association between hepatitis $\mathrm{C}$ infection and survival after orthotopic liver transplantation. Gastroenterology. 2002;122(4):889-896.

2. Demetris AJ. Evolution of hepatitis $\mathrm{C}$ virus in liver allografts. Liver Transpl. 2009;15(Suppl 2):S35-41.
3. Gallegos-Orozco JF, Yosephy A, Noble B, Aqel BA, Byrne TJ, Carey EJ, Douglas DD, et al. Natural history of post-liver transplantation hepatitis $\mathrm{C}$ : A review of factors that may influence its course. Liver Transpl. 2009;15(12):1872-1881.

4. Eghtesad B, Fung JJ, Demetris AJ, Murase N, Ness R, Bass DC, Gray EA, et al. Immunosuppression for liver transplantation in $\mathrm{HCV}$-infected patients: mechanismbased principles. Liver Transpl. 2005;11(11):1343-1352.

5. Kwo PY, Mantry PS, Coakley E, Te HS, Vargas HE, Brown R, Jr., Gordon F, et al. An interferon-free antiviral regimen for $\mathrm{HCV}$ after liver transplantation. N Engl J Med. 2014;371(25):2375-2382.

6. Charlton M. Telaprevir, boceprevir, cytochrome P450 and immunosuppressive agents--a potentially lethal cocktail. Hepatology. 2011;54(1):3-5.

7. Samuel D, Roche B. Ribavirin in the treatment of recurrent hepatitis $\mathrm{C}$ after liver transplantation: difficult to manage but essential for success. J Hepatol. 2007;46(6):988-991.

8. Hoofnagle JH, Sherker AH. Therapy for hepatitis C--the costs of success. N Engl J Med. 2014;370(16):1552-1553.

9. Kamal-Yanni M. Hepatitis C drug affordability. Lancet Glob Health. 2015;3(2):e73-74.

10. Banff schema for grading liver allograft rejection: an international consensus document. Hepatology. 1997;25(3):658-663.

11. Berenguer $\mathrm{M}$. What determines the natural history of recurrent hepatitis C after liver transplantation? J Hepatol. 2005;42(4):448-456.

12. Ishak K, Baptista A, Bianchi L, Callea F, De Groote J, Gudat F, Denk H, et al. Histological grading and staging of chronic hepatitis. J Hepatol. 1995;22(6):696-699.

13. Te HS. Recurrent hepatitis C: the bane of transplant hepatology. Hepatology. 2014;59(1):21-23.

14. Firpi RJ, Abdelmalek MF, Soldevila-Pico C, Cabrera R, Shuster JJ, Theriaque D, Reed AI, et al. One-year protocol liver biopsy can stratify fibrosis progression in liver transplant recipients with recurrent hepatitis $\mathrm{C}$ infection. Liver Transpl. 2004;10(10):1240-1247.

15. AASLD/IDSA/IAS-USA. Recommendations for testing, managing, and treating hepatitis C. http://www.hcvguidelines.org. Accessed April 24, 2015.

16. Hill A, Cooke G. Medicine. Hepatitis C can be cured globally, but at what cost? Science. 2014;345(6193):141-142.

17. Congly SE, Lee SS. Editorial: can we afford the new direct-acting antivirals for treatment of genotype 1 hepatitis C? Aliment Pharmacol Ther. 2014;40(8):983-984.

18. Valadao RM, Terrault NA. Older donors: mounting risks for the hepatitis $\mathrm{C}$-infected liver transplant recipient? Liver Transpl. 2009;15(7):677-681.

19. Rayhill SC, Wu YM, Katz DA, Voigt MD, Labrecque DR, Kirby PA, Mitros FA, et al. Older donor livers show early severe histological activity, fibrosis, and graft failure after liver transplantation for hepatitis C. Transplantation. 2007;84(3):331-339.

20. Wertheim JA, Petrowsky H, Saab S, Kupiec-Weglinski JW, Busuttil RW. Major challenges limiting liver transplantation in the United States. Am J Transplant. 2011;11(9):1773-1784. 